\title{
Prognostic Significance of Lymph Node Ratio After Resection of Peri-hilar Cholangiocarcinoma
}

\author{
Alfredo Guglielmi, Andrea Ruzzenete, Fabio Bagante, Tommaso Campagnaro, Simone Conci, \\ Calogero lacono
}

Department of Surgery, Division of General and Hepato-Pancreato-Biliary Surgery, University of Verona, Verona, Italy

\section{ABSTRACT}

Even though lymph node status is one of the most important predictors of survival for patients undergoing curative-intent surgery for peri-hilar cholangiocarcinoma (PHCC), the optimal lymph node staging system for PHCC has not been identified. Recently, the newly published 8th edition of the American Joint Committee on Cancer (AJCC) staging system reformed the criteria used to stage the lymph node status of PHCC patients but this change did not demonstrate to significantly improve the prognostic stratification of patients with PHCC. Among the several clinical tools that have been proposed in replacement of the AJCC $\mathrm{N}$ stages, the lymph node ratio (LNR) defined as the ratio of the number of metastatic lymph nodes to the number of lymph nodes harvested, demonstrated to improve the lymph node staging of patients with several gastrointestinal and hepatobiliary cancers. When tested in PHCC patients, while LNR was associated with patients' prognosis, the prognostic performances of LNR were strongly influenced by the number of lymph node harvested suggesting that LNR might be of less clinical value in PHCC patients due to the relatively small number of lymph node harvested compared with other gastrointestinal cancers. While other clinical tool (i.e. LODDS) has been proposed to assess lymph node status, further studies are needed to improve the lymph nodal staging of PHCC patients.

Key words: liver surgery, peri-hilar cholangiocarcinoma, lymphadenectomy, lymph-node ratio

\section{INTRODUCTION}

With about 12,000 new cases per year and an incidence of 1-2 per 100,000 individuals in the United States, perihilar cholangiocarcinoma (PHCC) is the most frequent form of cholangiocarcinoma accounting for $60-70 \%$ of bile duct cancers (1-4). PHCC can be an aggressive malignancy with low incidence $(\approx 50 \%)$ of patients presenting with a resectable disease at the time of diagnosis and a disappointing prognosis even after curative-intent surgery (5-year overall survival ranging from 20 to 42\%) (5-7). While several factors has been associated with the survival of patients who underwent curative intent resection of PHCC
Corresponding author:

Prof. Calogero lacono, MD, Department of Surgery and Oncology Division of General and HepatoPancreato-Biliary Surgery,

School of Medicine, University of Verona P. Ie L.A. Scuro, 37134 Verona, Italy Tel. + 390458124412 Fax + 390458027426 E-mail: Calogero.lacono@univr.it

Received: 18.10 .2018

Accepted: 03.12.2018

Copyright () Celsius Publishing House www sgo-iasgo.com 
including tumor extension, vascular invasion, margin status, grade of tumor differentiation, and CA19-9 serum level, lymph-node status is the strongest predictors of prognosis with a 5 -year overall survival of only $10-20 \%$ among patients with metastatic lymphnodes (N1 disease) (2, 8-11). For this reason, several classification and mathematical computational clinical tools have been proposed to stage the lymph-node status of PHCC patients to better identify the subset of patients with a high risk of recurrence after surgery and who might benefit from aggressive perioperative chemoradiotherapy treatments.

\section{LYMPHADENECTOMY AND LYMPH NODE STAGING}

The lymph node drainage for the liver includes a superficial lymphatic pathway under the Glisson's capsule of the liver and a deep lymphatic drainage that following the portal veins drains into the lymph nodes at the hilum of the liver. The superficial lymphatic pathway include three major groups: 1) the most common lymph node basins site of metastasis through the hepatoduodenal and gastro-hepatic ligament pathway, 2) the diaphragmatic lymphatic plexus where the liver is directly in contact with the diaphragm with the liver bare area and indirectly through the coronary and triangular ligaments, and 3) lymphatic plexus along the falciform ligament to the deep superior epigastric node in the anterior abdominal wall along the deep superior epigastric artery below the xiphoid cartilage. Moreover, the deep lymphatic drainage includes two major lymph node chains in the hepatoduodenal ligament: 1) the hepatic artery chain that follows the common hepatic artery to the lymph node at the celiac axis and then into the cisterna chyli 2) the posterior periportal chain, located posterior to the portal vein in the hepatoduodenal ligament that drains into the retropancreatic nodes and the aortocaval node into the cisterna chyli and the thoracic duct (12).

Lymphadenectomy for PHCC is usually performed during the hepatic pedicle dissection and should include the dissection of regional lymph nodes including hilar (basin \#12h), peri-choledochal (\#12b and \#12c), peripancreatic (\#13a), periportal (\#12p), and common hepatic artery (\#8a and \#8p) nodes (13). Even though periaortic lymph nodes might be included in an extended lymphadenectomy, several authors have reported that curative intent surgery should be performed only on patients without distant lymph node metastasis (negative periaortic lymph node) (13). In a systematic review on the lymph node dissection in resectable PHCC, Kambakamba et al. reported a median number of lymph node harvested of 7 widely ranging from 2 to 24 lymph nodes and the retrieval of 3 lymph nodes was the most commonly reported median lymph node (14). While the median number of lymph node harvested was not associated with the incidence of lymph node metastasis (N1 patients) and with patients' survival, in a sub-analysis, the studies reporting a median number of lymph nodes harvested between 7 and 9 reported the highest incidence of $\mathrm{N} 1$ patients (14). The American Joint Committee on Cancer (AJCC) staging system is the most used clinical tool used to prognostically stratify PHCC patients (15). Even though AJCC suggested a minimum number of harvested lymph nodes for an adequate lymph node staging of patients with intrahepatic cholangiocarcinoma, the number of lymph nodes harvested for PHCC patients is still under debate (15). Moreover, the newly published AJCC 8th edition significantly changed the criteria for the nodal staging of patients with PH-CCA. While in the AJCC 7th edition the location of metastatic lymph nodes was used to define the $\mathrm{N}$ stage for PHCC patients (N1, regional LN metastasis; N2, peri-aortic, peri-caval, superior mesenteric artery, and/or celiac artery LN metastasis), the AJCC 8th edition identify the number of metastatic lymph node as the most accurate variable associated with patients' prognosis defining stage $\mathrm{N} 1$ as patients with 1-3 metastatic lymph nodes and stage N2 as patients with $>3$ lymph nodes $(15,16)$.

\section{LYMPH NODE RATIO}

Even though in a recent analysis of 214 patients who underwent surgery for PH-CCA, Ruzzenente et al. reported that the AJCC 8th edition $\mathrm{N}$ staging performed better than the 7th edition, the AJCC lymph node staging has a poor ability to predict the prognosis of patients undergoing liver resection for PH-CCA (c-index $<0.6$ ) and the optimal $\mathrm{N}$ staging for PH-CCA has not been identified (17).

Lymph node ratio (LNR) defined as the ratio of the number of metastatic lymph nodes to the number of lymph nodes harvested, demonstrated to improve the lymph node staging of patients with gastrointestinal and hepatobiliary cancers including pancreatic ductal adenocarcinoma, ampulla of Vater, distal bile duct cancer, and intrahepatic cholangiocarcinoma (18-21).

Guglielmi et al. reported the prognostic value of the lymph node status, total number of lymph node evaluated and LNR of 62 patients with PHCC who underwent surgical resection with curative intent (22). With a median number of harvested lymph nodes of 7 , 
median overall survival was 42 months for NO patients compared with 23 months for N1 patients ( $p$-value $=0.03$ ). Moreover, median overall survival increased from 3 to 19 and 29 months for patients with $0,1-3$ and $>3$ harvested lymph nodes, respectively ( $p$-value $<0.01)$. The authors identified a cut-off for LNR of 0.25 reporting a 5 -year overall survival of 0 and $23 \%$ for patients with $>0.25$ and $\leq 0.25$ LNR, respectively ( $p$-value $=0.03$ ) (22).

The group of the Nagoya University investigated the prognostic relevance of LNR analyzing long-term outcomes of 320 consecutive patients with PHCC who underwent surgery from January 2000 to December 2009 (23). The author reported a 5-year overall survival of $60 \%$ for NO patients compared with $19 \%$ for N1 patients. Lymph node ratio (LNR) was analyzed in the 146 patients who had lymph node metastasis and the survival for patients with an LNR of 0.2 or less was significantly better than that for patients with an LNR greater than 0.2 (5-year OS: LNR $\leq 0.2,21 \%$ vs $L N R>0.2$, $13 \%, p$-value $=0.032$ ) while the difference in survival was not statistically different when the LNR cutoff value was set at 0.25 or 0.3 . Of note, in the multivariable model, LNR did not result an independent predictor of survival for PHCC patients (23). The authors concluded their article considering that while LNR might be used to minimize the inconsistency and variability of nodal assessment in an effort to standardize the lymph node staging, LNR is largely influenced by the number of lymph nodes harvested and that LNR in PHCC patients might be of less clinical value due to the relative small number of lymph node harvested compared with other gastrointestinal cancers (23).

Hakeem et colleague investigated the long-term outcome of 78 patients who underwent surgery for PHCC at the Leeds Teaching Hospitals in the UK between 1994 and 2010 (24). The authors reported that while the 5-year OS resulted $10 \%$ and $41 \%$ for patients with and without lymph node metastasis $(p<0.001)$, a LNR $>0.37$ was an independent predictors of disease free survival (DFS) but was not associated with patients' overall survival (24).

Oshiro et al. retrospectively analyzed a total of 60 consecutive patients who underwent resection for extrahepatic cholangiocarcinoma at the Department of Surgery of the University of Tsukuba in Japan between January 2001 and December 2009 (25). According with previous data present in the literature the authors used 0.2 as the curt-off for LNR reporting $56 \%(n=34)$ of patients with negative lymph nodes and $22 \%(n=13)$ and $22 \%(n=13)$ of patients had LNR $\leq 0.2$ and $>0.2$, respectively. While no patients with $L N R>0.2$ survived five years after surgery, NO patents had a 5-year OS of
$44 \%$, compared with $10 \%$ for patients with LNR $\leq 0.2$ ( $p=0.023$ ). Moreover, LNR resulted associated with patients' prognosis in both the univariate $(p=0.016)$ and multivariable $(p=0.022)$ analyses (25).

Mao et colleagues performed an analysis of the American population-based Surveillance, Epidemiology, and End Results (SEER) database to investigate the prognostic role of the patterns of lymph node dissection for patients who underwent surgery for PHCC between 1998 and 2008 (26). The authors reported that among the 1,116 patients with at least one harvested lymph node, the largest difference in terms of overall survival was identified when 0.27 was choose as a cutoff value for LNR. Patients with a LNR $\leq 0.27$ had a 5 -year OS of $19 \%$ compared with only $9 \%$ for patient with a LNR $>0.27$ $(p=0.001)(26)$.

Bagante et al. assessed the prognostic performance of different nodal staging in an international multiinstitutional database including 437 patients who underwent surgery with curative intent for PHCC between 1995 and 2014 (27). According with the results of the group of Nagoya, the authors reported that the prognostic performance of LNR was strongly influenced by the number of lymph node harvested. In particular, while LNR and AJCC $\mathrm{N}$ stage had similar performance among patients with $<4$ lymph node harvested, LNR performed better at determining prognosis among patients with $\geq 4$ lymph node harvested compared with the AJCC nodal staging (27).

\section{OTHER METHODS TO PROGNOSTICALLY ASSESS LYMPH NODE STATUS}

The limits of LNR for PHCC patients have been also underlined by Conci et al. who recently compared the different approaches used to stage the lymph node status of patients who underwent hepatectomy for PHCC at the University of Verona between September 1990 and December 2014 (28). The mathematical transformation of the ratio between the number of lymph nodes harvested and the number of metastatic lymph nodes (log odds of metastatic lymph nodes, LODDS) was the factor with the highest area under the receiver operating characteristic curve (AUC) predicting the 3-year OS (AUC $=0.71$ ) compared with lymph node ratio ( $L N R, A U C=0.60$ ), number of metastatic lymph node $(A \cup C=0.59)$, and AJCC 7th edition N-staging (AUC $=0.54$ ) (28). The authors determined that while the mathematical transformation of number of metastatic lymph node and number of harvested lymph nodes as LNR and LODDS are better predictors of survival than AJCC $\mathrm{N}$ staging, LODDS resulted the 


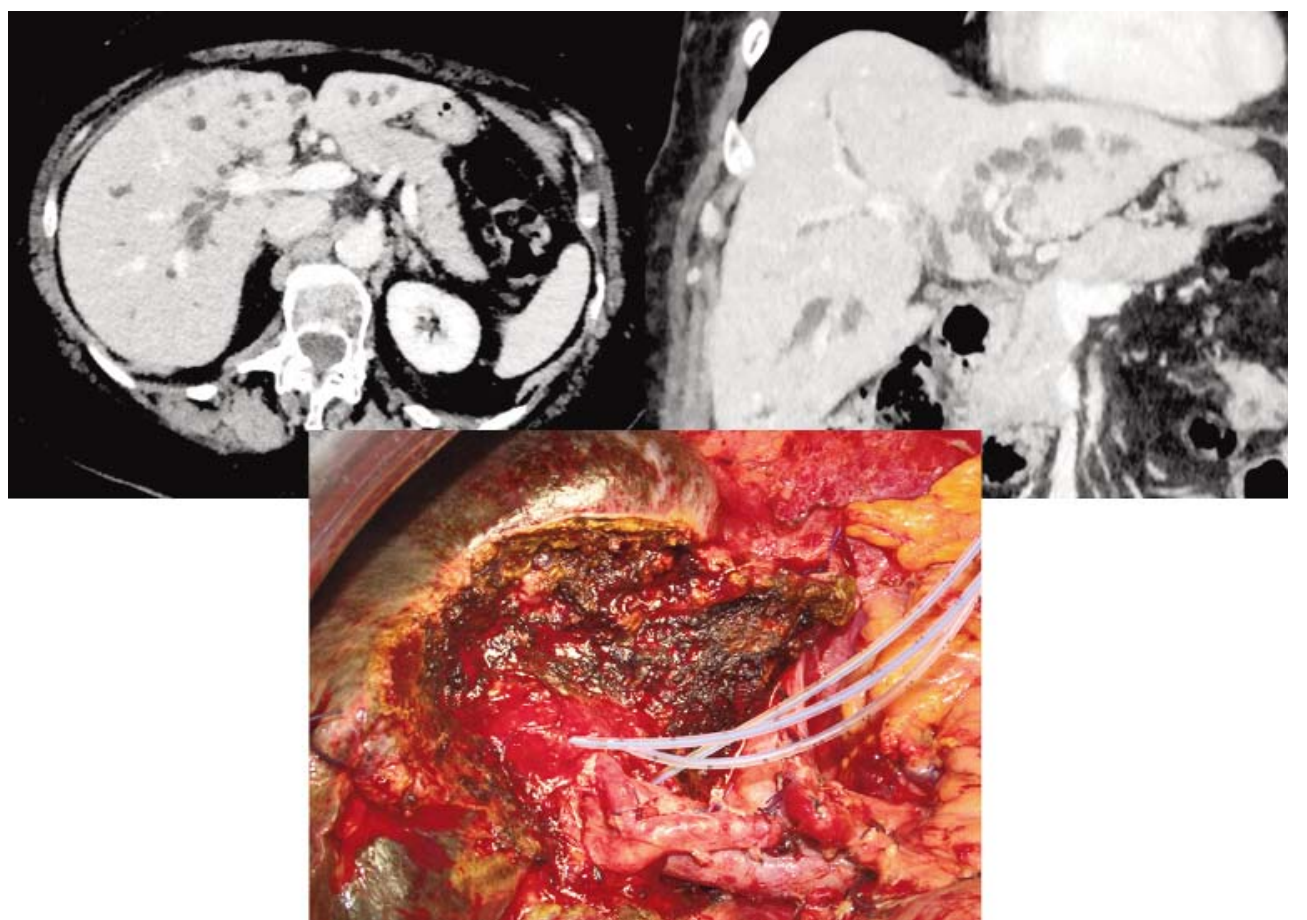

Figure 1 - a) axial and b) coronal CT scan of a peri-hilar cholangiocarcinoma type Illb according with Bismuth-Corlette classification, and c) intraoperative aspect of left hepatectomy, bile duct resection, and lymphadenectomy

most accurate and predictive lymph node staging for patients undergoing curative intent surgery for PHCC.

\section{CONCLUSION}

Even though there is a general consent on the benefit of lymphadenectomy for PHCC patients, the exact number of harvested lymph node has not been found. While LNR has been associated with the prognosis of patients undergoing curative intent surgery for PHCC, the best cut-off to discriminate patients with favorable and poor prognosis among patients with positive lymph nodes has not been clearly found. Moreover, several authors including our group have reported that, LNR for PHCC patients might be of less clinical value due to the relatively small number of lymph node harvested compared with other gastrointestinal cancers while different mathematical estimation (i.e. LODDS) might improve the lymph node staging of PHCC patients. Even though lymph node status is the most important factors associated with PHCC patients' prognosis, the optimal lymph node staging has not been identified. Given the low incidence of PHCC, multi-institutional studies are needed to provide consistent evidence on the $\mathrm{N}$ staging for PHCC patients.
Funding / conflict of interest: None.

\section{REFERENCES}

1. Nakeeb A, Pitt HA, Sohn TA, Coleman J, Abrams RA, Piantadosi S, et al. Cholangiocarcinoma. A spectrum of intrahepatic, perihilar, and distal tumors. Ann Surg. 1996;224(4): 463-73; discussion 473-5.

2. DeOliveira ML, Cunningham SC, Cameron JL, Kamangar F, Winter $\mathrm{JM}$, Lillemoe KD, et al. Cholangiocarcinoma: thirty-one-year experience with 564 patients at a single institution. Ann Surg. 2007; 245(5):755-62.

3. Siegel RL, Miller KD, Jemal A. Cancer statistics, 2015. CA Cancer J Clin. 2015;65(1):5-29.

4. Siegel RL, Miller KD, Jemal A. Cancer statistics, 2018. CA Cancer J Clin. 2018;68(1):7-30.

5. Choi JY, Kim MJ, Lee JM, Kim KW, Lee JY, Han JK, et al. Hilar cholangiocarcinoma: role of preoperative imaging with sonography, MDCT, MRI, and direct cholangiography. AJR Am J Roentgenol. 2008;191(5):1448-57.

6. Ruys AT, van Beem BE, Engelbrecht MR, Bipat S, Stoker J, Van Gulik TM. Radiological staging in patients with hilar cholangiocarcinoma: a systematic review and meta-analysis. Br J Radiol. 2012;85(1017): $1255-62$.

7. Suarez-Munoz MA, Fernandez-Aguilar JL, Sanchez-Perez B, PerezDaga JA, Garcia-Albiach B, Pulido-Roa Y, et al. Risk factors and classifications of hilar cholangiocarcinoma. World J Gastrointest Oncol. 2013;5(7):132-8.

8. Kitagawa Y, Nagino M, Kamiya J, Uesaka K, Sano T, Yamamoto H, et al. Lymph node metastasis from hilar cholangiocarcinoma: audit of 110 patients who underwent regional and paraaortic node dissection. Ann Surg. 2001; 233(3):385-92.

9. Lee SG, Song GW, Hwang S, Ha TY, Moon DB, Jung DH, et al. Surgical treatment of hilar cholangiocarcinoma in the new era: the 
Asan experience. J Hepatobiliary Pancreat Sci. 2010;17(4):476-89.

10. Song SC, Choi DW, Kow AW, Choi SH, Heo JS, Kim WS, et al Surgical outcomes of 230 resected hilar cholangiocarcinoma in a single centre. ANZ J Surg. 2013; 83(4):268-74.

11. Furusawa N, Kobayashi A, Yokoyama $\mathrm{T}$, Shimizu A, Motoyama $\mathrm{H}$, Miyagawa S. Surgical treatment of 144 cases of hilar cholangiocarcinoma without liver-related mortality. World J Surg. 2014;38(5): 1164-76.

12. MG H. Atlas of Lymph Node Anatomy. 2013.

13. Miyazaki M, Ohtsuka M, Miyakawa S, Nagino M, Yamamoto M, Kokudo N, et al. Classification of biliary tract cancers established by the Japanese Society of Hepato-Biliary-Pancreatic Surgery: 3(rd) English edition. J Hepatobiliary Pancreat Sci. 2015;22(3):181-96.

14. Kambakamba P, Linecker M, Slankamenac K, DeOliveira ML. Lymph node dissection in resectable perihilar cholangiocarcinoma: a systematic review. Am J Surg. 2015;210(4):694-701.

15. Amin MB, American Joint Committee on Cancer, American Cancer Society. AJCC cancer staging manual. Chicago IL: American Joint Committee on Cancer; Springer 2017.

16. Compton CC, American Joint Committee on Cancer. AJCC cancer staging atlas : a companion to the seventh editions of the AJCC cancer staging manual and handbook. In Second edition / Edition. New York: Springer, 2012.

17. Ruzzenente A, Bagante F, Ardito F, Campagnaro T, Scoleri I, Conci S, et al. Comparison of the 7th and 8th editions of the American Joint Committee on Cancer Staging Systems for perihilar cholangiocarcinoma. Surgery. 2018;164(2): 244-250.

18. Inoue $\mathrm{K}$, Nakane $\mathrm{Y}$, liyama $\mathrm{H}$, Sato M, Kanbara T, Nakai K, et al. The superiority of ratio-based lymph node staging in gastric carcinoma. Ann Surg Oncol. 2002;9(1):27-34.

19. Pawlik TM, Gleisner AL, Cameron JL, Winter JM, Assumpcao L, Lillemoe KD, et al. Prognostic relevance of lymph node ratio following pancreaticoduodenectomy for pancreatic cancer. Surgery. 2007;141(5):610-8.

20. Falconi M, Crippa S, Domínguez I, Barugola G, Capelli P, Marcucci S, et al. Prognostic relevance of lymph node ratio and number of resected nodes after curative resection of ampulla of Vater carcinoma. Ann Surg Oncol. 2008; 15(11):3178-86.

21. Tamandl D, Kaczirek K, Gruenberger B, Koelblinger C, Maresch J, Jakesz $\mathrm{R}$, et al. Lymph node ratio after curative surgery for intrahepatic cholangiocarcinoma. Br J Surg. 2009;96(8):919-25.

22. Guglielmi A, Ruzzenente A, Campagnaro T, Pachera S, Conci S, Valdegamberi $A$, et al. Prognostic significance of lymph node ratio after resection of peri-hilar cholangiocarcinoma. HPB (Oxford). 2011;13(4):240-5.

23. Aoba T, Ebata T, Yokoyama Y, Igami T, Sugawara G, Takahashi Y, et al. Assessment of nodal status for perihilar cholangiocarcinoma: location, number, or ratio of involved nodes. Ann Surg. 2013; 257(4):718-25

24. Hakeem AR, Marangoni G, Chapman SJ, Young RS, Nair A, Hidalgo $\mathrm{EL}$, et al. Does the extent of lymphadenectomy, number of lymph nodes, positive lymph node ratio and neutrophil-lymphocyte ratio impact surgical outcome of perihilar cholangiocarcinoma? Eur $\mathrm{J}$ Gastroenterol Hepatol. 2014;26(9):1047-54.

25. Oshiro Y, Sasaki R, Kobayashi A, Murata S, Fukunaga K, Kondo T, et al. Prognostic relevance of the lymph node ratio in surgical patients with extrahepatic cholangiocarcinoma. Eur J Surg Oncol. 2011; 37(1):60-4.

26. Mao K, Liu J, Sun J, Zhang J, Chen J, Pawlik TM, et al. Patterns and prognostic value of lymph node dissection for resected perihilar cholangiocarcinoma. J Gastroenterol Hepatol. 2016; 31(2):417-26.

27. Bagante F, Tran T, Spolverato G, Ruzzenente A, Buttner S, Ethun CG, et al. Perihilar Cholangiocarcinoma: Number of Nodes Examined and Optimal Lymph Node Prognostic Scheme. J Am Coll Surg. 2016; 222(5):750-759.e2.

28. Conci S, Ruzzenente A, Sandri M, Bertuzzo F, Campagnaro T, Bagante $F$, et al. What is the most accurate lymph node staging method for perihilar cholangiocarcinoma? Comparison of UICC/ AJCC pN stage, number of metastatic lymph nodes, lymph node ratio, and log odds of metastatic lymph nodes. Eur J Surg Oncol. 2017;43(4):743-750. 